\title{
Antidiabetic drug treatment confounds gut dysbiosis associated with type 2 diabetes mellitus
}

Patients with type 2 diabetes mellitus (T2DM) have been reported to have alterations in the composition and function of their gut microbiota, which is suggestive of a microbe-mediated mechanism of disease. However, few studies reporting this correlation have taken into account the antidiabetic medications used by patients, which could themselves affect the gut microbiota.

A new study published in Nature by Forslund et al. shows that metformin (the most commonly prescribed drug for lowering blood glucose levels in patients with T2DM) distorts the gut microbiota signature associated with T2DM. The findings highlight the need for caution in interpreting both studies correlating the gut microbiota to chronic disease and studies of faecal transplantation that reproduce disease phenotypes to investigate microbe-mediated disease mechanisms. "Alterations in the gut microbiota might not be features characteristic of specific disorders themselves," explains lead investigator Oluf Pedersen. "Rather, the intrinsic gut microbiota imbalances associated with a given disorder might be masked or confounded by patients' treatment regimens."

The researchers sequenced faecal DNA samples collected from 383 Danish individuals (75 with T2DM, 31 with type 1 diabetes mellitus and 277 nondiabetic controls), who were participants in the Metagenomics of the Human Intestinal Tract (MetaHIT) project. Combining these results with those of two previous metagenomic studies of
T2DM (a Swedish study of 53 female patients with T2DM and 92 nondiabetic individuals and a Chinese study of 71 patients with T2DM and 185 nondiabetic controls) enabled the team to assemble a multi-country T2DM metagenomic dataset of 784 gut metagenomes for taxonomic and functional profiling. Treatment history was available for participants in all cohorts. Initial analysis (unstratified for treatment) comparing gut microbial taxonomic and functional profiles between T2DM $(n=199)$ and control $(n=554)$ metagenomic samples revealed many genus-level associations; however, these associations were not consistently observed in samples from all three studies.

Believing confounding treatments to be responsible for the inconsistency in the inferred associations, the researchers stratified samples on the basis of patients' diet and antidiabetic medication (metformin, insulin, statins and drugs to lower blood pressure). However, only metformin treatment altered the composition and function of the gut microbiota. Patients with T2DM who were treated with metformin $(n=93)$ had considerably more Escherichia spp. and less Intestinibacter spp. than untreated patients with T2DM $(n=106)$; a finding confirmed in an independent cohort of patients with T2DM. As Escherichia spp. have the potential to produce short-chain fatty acids such as butyrate and propionate, which can trigger intestinal gluconeogenesis via complementary mechanisms and thus lower blood glucose levels, the findings support the theory that the gut microbiota contributes to the beneficial glycaemic effects of metformin. Furthermore, metformin treatment was associated with compositional and functional microbiome alterations (increased abundance of Escherichia spp. and enrichment of virulence factors and genes encoding proteins involved in gas production, respectively) that are consistent with the adverse gastrointestinal effects reported in $\sim 30 \%$ of patients with T2DM who take the drug.

Although the results suggest that both the therapeutic and adverse effects of metformin are partially mediated by the gut microbiota, the authors acknowledge in their report that further studies are required to conclude causality and to determine the mechanisms underlying this mediation.

\section{David Holmes}
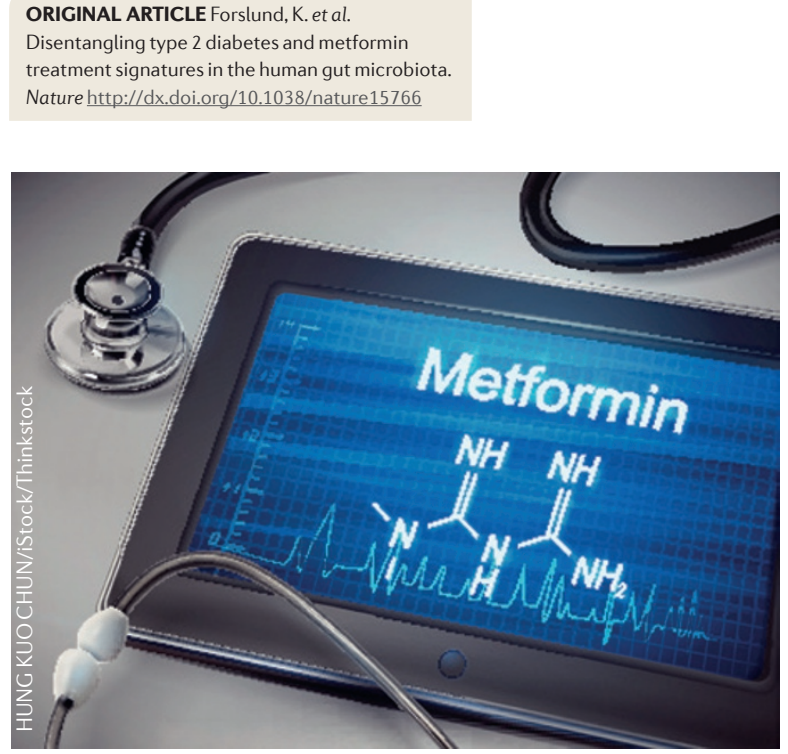